\title{
Verification on Gradual Escalation Phenomenon of Violation Behavior-Using an Experimental Paradigm for Risk Diversification
}

\begin{abstract}
Atsuo Murata
Okayama University, Okayama, Japan

Gradual violation of a safety rule instead of violating a safety rule at a time paralyzes our ethical attitude towards safety and makes us less guilty of violating a safety rule or ethics. Gradual escalation of risk taking behavior dilutes more our attitude towards safety and makes us underestimate a risk than taking a risk at a time. In other words, it should be empirically verified whether the diversification of risk promotes the risk seeking behavior as compared with the risk taking at a time. It is possible that such a gradual escalation of risk taking behavior leads to a crucial accident like JCO (Japan Nuclear Fuel Conversion Office) critical mass accident. Thus, we paid attention to unethical behavior, in particular, the (intentional) violation of rules motivated by the economic incentive or the pursuit of efficiency. When we face a situation under which we must weigh the ethics of safety rule and the efficiency (economic aspect) in the balance, we may pursuit efficiency by taking a risk gradually by diversifying the risk. More concretely, it was hypothesized that we tend to underestimate the total risk when the total risk is divided to a few risk-taking behaviors than when the total risk is taken at a time. As a result of exploring the relationship between the diversification of risk (accepting (taking) the total risk gradually by dividing it into a few trials) and the risk assessment, it was clarified that we tended to underestimate the total risk when the total risk was divided into a few risk-taking behaviors than when the total risk was taken at a time.
\end{abstract}

Keywords: diversification of risk, slippery-slope effect, gradual escalation of violation behavior, abrupt commission of violation behavior, safety management

\section{Introduction}

It has been pointed out that organizational managers and leaders are willing to abandon ethical standard in the face of economics incentives (Hosmer, 1987). Moore, Tetlock, Tanlu, and Bazerman (2006) showed that accounting firms must provide independent financial statements and report clients' mismanagement at the risk of displeasing their clients and losing lucrative service contracts. In such a situation, we tend to select the violation of social rules or norms and pursue economic incentives.

In the field of behavioral ethics, it has been shown that we tend to overlook others' unethical behavior when it occurs gradually than when it occurs at a burst (Gino \& Bazerman, 2009; Bazerman \& Tenbrunsel, 2012). We are less likely to take others unethical behavior seriously when it eroded gradually over time rather

Atsuo Murata, Ph.D., professor, Department of Intelligent Mechanical Systems, Graduate School of Natural Science and Technology, Okayama University. 
than when it eroded in one gulp. Such a phenomenon is known as slippery-slope effect or gradual escalation of unethical behavior. Slippery-slope effect can be defined as follows: a process or series of events that are hard to stop or control once it has begun and that usually leads to worse or more difficult things, or a course of action that seems to lead inevitably from one action or result to another with unintended consequences. In such a situation, we tend to weigh the ethicality and the economic incentive in the balance, and gradually escalate unethical behaviors.

Even in safety management, we are forced to be confronted with such a situation where we weigh the safety (ethicality) and the efficiency (economic incentive) in the balance. Although we thoroughly understand that it is essential to observe safety rules, we gradually escalate and erode safety ethics or rules and pursuit efficiency. We feel less guilty when violating a safety rule gradually than when violating a safety rule at a time. Such a slippery-slope effect leads to a blind spot of safety, and unintended crucial accidents like critical mass accident at the uranium processing plant of JCO Tokai Works Test Facility (Chiles, 2002).

The slippery-slope effect pointed out by Gino and Bazerman (2009) can also be interpreted from the viewpoint of risk taking. Gradual violation of a safety rule instead of violating a safety rule at a time paralyzes our ethical attitude towards safety and makes us less guilty of violating a safety rule or ethics. Gradual escalation of risk taking behavior dilutes more our attitude towards safety and makes us underestimate a risk further than taking a risk at a time. In other words, it should be empirically verified whether the diversification of risk promotes the risk seeking behavior as compared with the risk taking at a time.

Thus, it is possible that such a gradual escalation of risk taking behavior leads to a crucial accident like JCO critical mass accident. We paid attention to unethical behavior, in particular, the (intentional) violation of rules motivated by the economic incentive or the pursuit of efficiency.

When we face a situation under which we must weigh the ethics of safety rule and the efficiency (economic aspect) in the balance, we may pursuit efficiency by taking a risk gradually by diversifying the risk. More concretely, it was hypothesized that we tend to underestimate the total risk when the total risk is divided to a few risk-taking behaviors than when the total risk is taken at a time. We frequently suffer from and are got into the trap of cognitive illusions (biases) (Kahneman, 2011; Tversky \& Kahneman, 1974; Kahneman \& Tversky, 1984; Altman, 2012; Anger, 2012; Ariery, 2009, 2010, 2012; Bazerman \& Moore, 2001), and it has been pointed out that falling into the trap of such cognitive biases can be a trigger of crucial accidents (Murata \& Nakamura, 2014). It is important to identify our behavioral characteristics that the diversification of risk affects our feeling about the risk taking behavior, and the gradual risk taking makes us feel less unethical and guilty of risk taking although the risk is the same in spite of taking it gradually or at a time.

In this study, an attempt was made to verify such a cognitive bias related to the diversification of risk. Exploring the relationship between the diversification of risk (accepting (taking) the total risk gradually by dividing it into a few trials) and the risk assessment, we empirically verified the tendency to underestimate the total risk when the total risk was divided into a few risk-taking behaviors than when the total risk was taken at a time. Some implications for safety management were given from the perspective of diversification of risk.

\section{Method}

\section{Participants}

Forty eight graduate or undergraduate students from 22 to 24 years old took part in the experiment. All agreed with the participation in the questionnaire survey and receiving a brief explanation on the survey. 


\section{Task, Design, and Procedure}

This questionnaire survey was carried out to verify the hypothesis that the diversification of risk will mitigate the risk and lead to under-evaluation of risk. The following two questions A and B were prepared, and each participant was required to select one of the two alternatives 1 or 2 .

(A) Assume that you are a mountaineer, and that you cannot descend a mountain safely without committing a risk. There are two ways of committing a risk. One is to descend a mountain (taking a risk) without stopping by taking into account the change of weather and the exhaustion of bodily power. Although this may be a good method and increase the probability of success, this, on the contrary, may induce the danger of life. Another is to descend a mountain gradually and diversifying the total risk. Which alternative do you choose?

(1) Descend a mountain (taking a risk) without stopping.

(2) Descend a mountain gradually (diversifying the total risk).

(B) Assume that you must pay back your debts of $\$ 1,000$. If you don't pay this back, the loan shark is strict in the collection of debts. As you are working at the accounting department of your company, you are planning to falsify the company's accounting temporarily, pay back the debts using the company's funds, and make up for this fund later. If you falsify the company's accounting at once, the dishonest accounting may be readily revealed. There are two ways to falsify the company's accounting temporarily. Which alternative do you feel is safer and choose?

(1) Falsify the company's accounting at once.

(2) Diversify the risk by 10 times, and falsify the company's accounting by $\$ 100$.

\section{Results and Discussion}

As shown in Figure 1, we hypothesized that we feel less risk as we diversify risk. In Figure 2, the percentage of each alternative for the questionnaire (A) is compared. As a test for two proportions, a significant difference $(z=2.43, p<0.05)$ was detected. A similar result for the questionnaire (B) is summarized in Figure 3. A similar test for two proportions revealed a marginally significant difference $(z=1.63, p=0.074)$.

From Figure 2 (a problem on descending a mounting under a risky situation), the percentage of each alternative for the alternative (2) is larger than that of the alternative (1). From Figure 3 (a problem on a cheating from accounting department), the alternative (2) tended to be selected more frequently than the alternative (1). From these results, as hypothesized in Figure 1, one seems to feel less risky as we take a risk gradually and diversify it.

Such a psychological property leads to the illegal six times uranium processing alteration of JCO critical mass accident (see Figure 4). In this plant, the observance of limitation of mass and limitation of shape was strictly imposed on the manufacturing process of uranium solution by Science and Technology Agency, Japan. In spite of this, the manufacturing process was illegally altered over six times as shown in Figure 4. This must correspond to the gradual erosion of safety rule. Therefore, such a property experimentally clarified in this experiment should be exploited in risk management strategies. As this example demonstrates, feeling less risky by the diversification of risk must be an important human property, and paralyze unconsciously our attitude to safety. The result tells a crucial factor in risk management. Violation of rules by diversifying risk feels us less risky might lead to a crucial accident like JCO critical mass accident. 


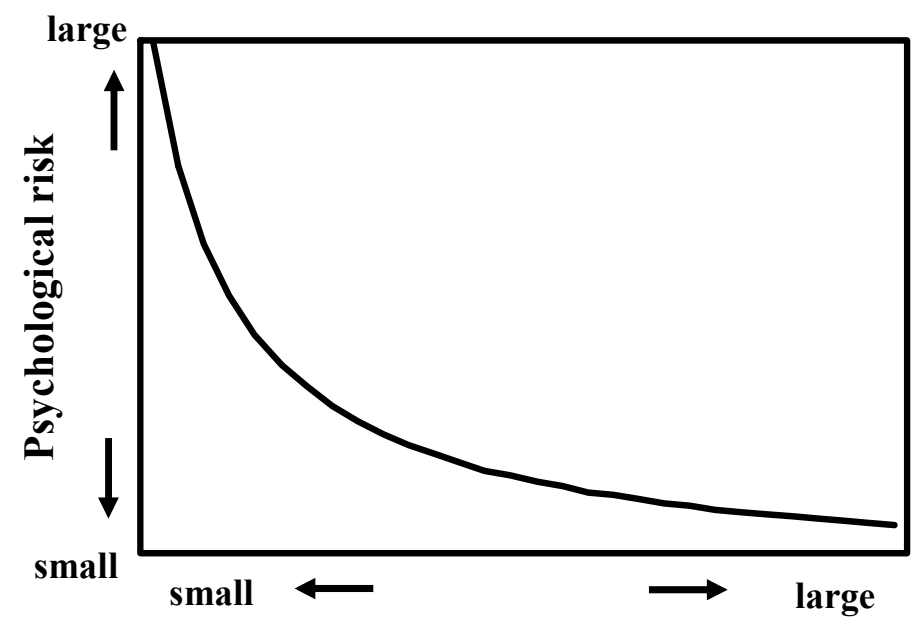

Number of risk diversification

Figure 1. Hypothetical relation between number of risk diversification and psychological risk. The hypothesis is that risk diversification makes us feel less risky.

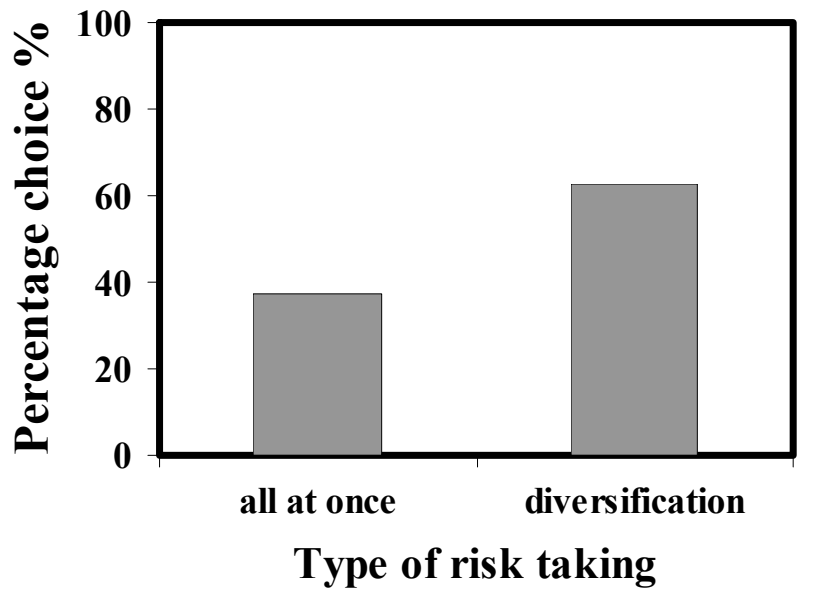

Figure 2. Percentage of each alternative for the questionnaire (1).

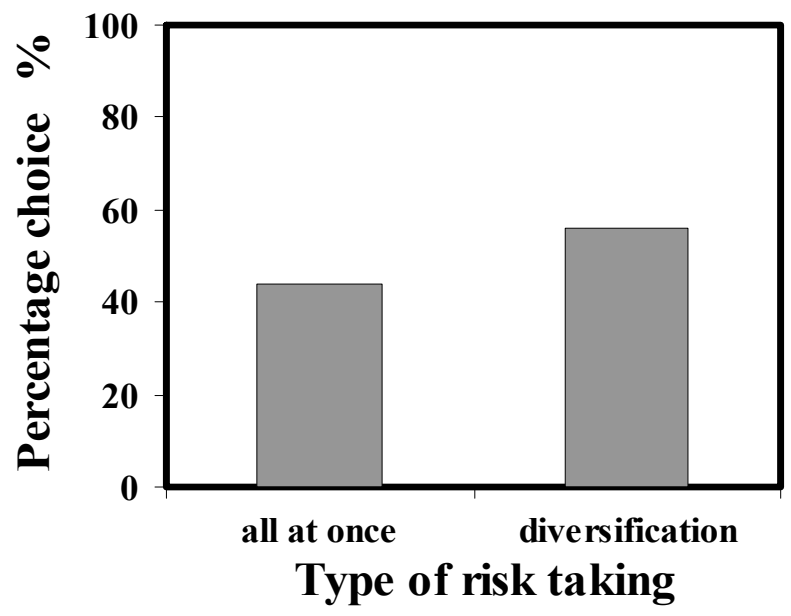

Figure 3. Percentage of each alternative for the questionnaire (2). 
Original manufacturing process specified by Science and Technology Agency

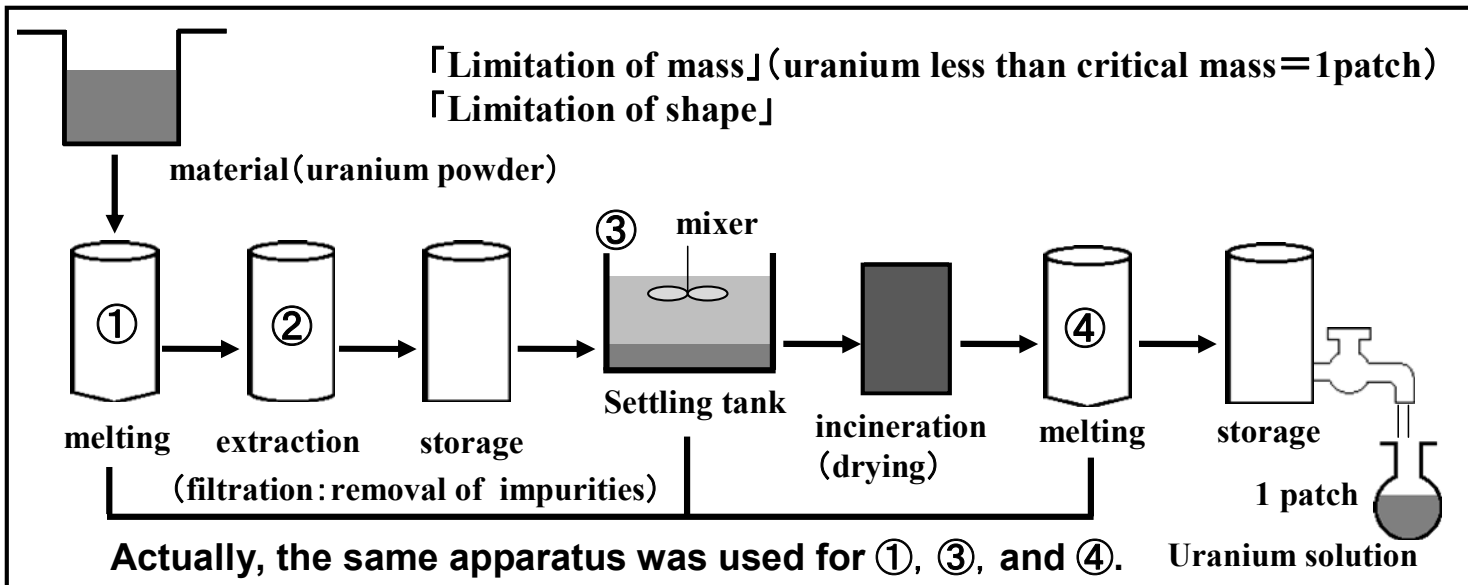

1st alteration of manufacturing process

(1) (2) (3) $\square$ Jobs (1), (2), and (3) were carried out simultaneously and parallel.

2nd alteration of manufacturing process

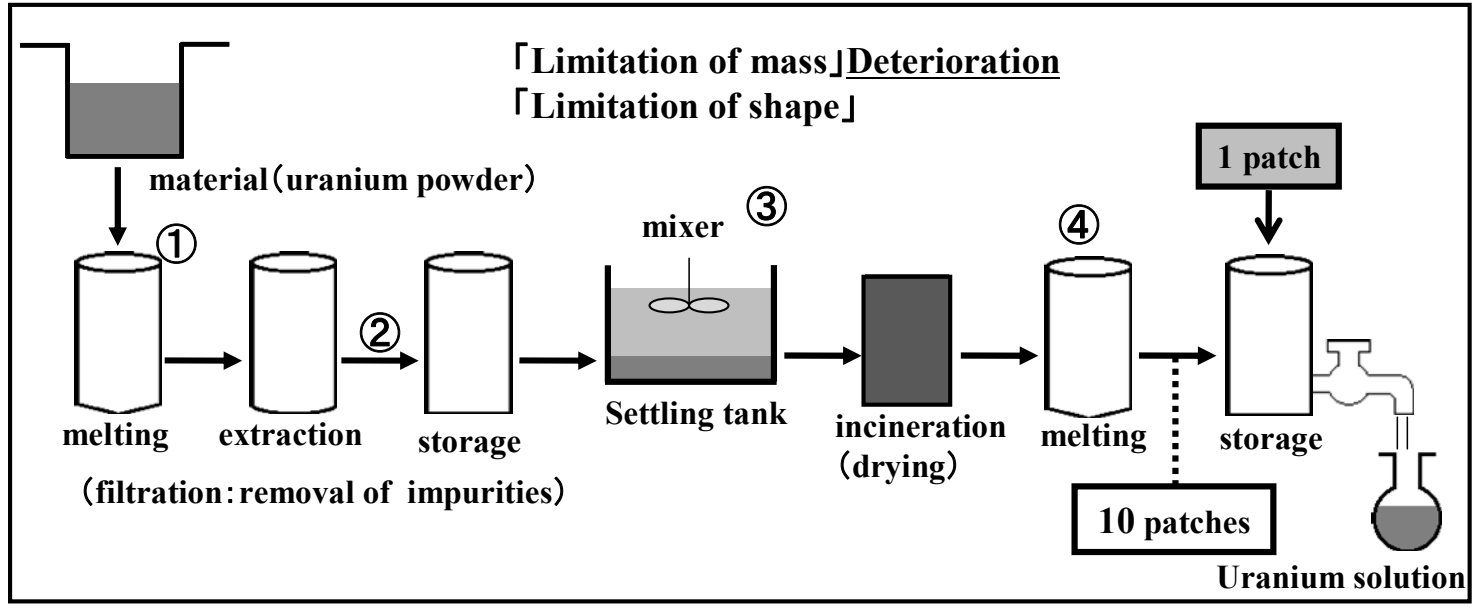

(Figure 4 to be continued) 
3rd alteration of manufacturing process

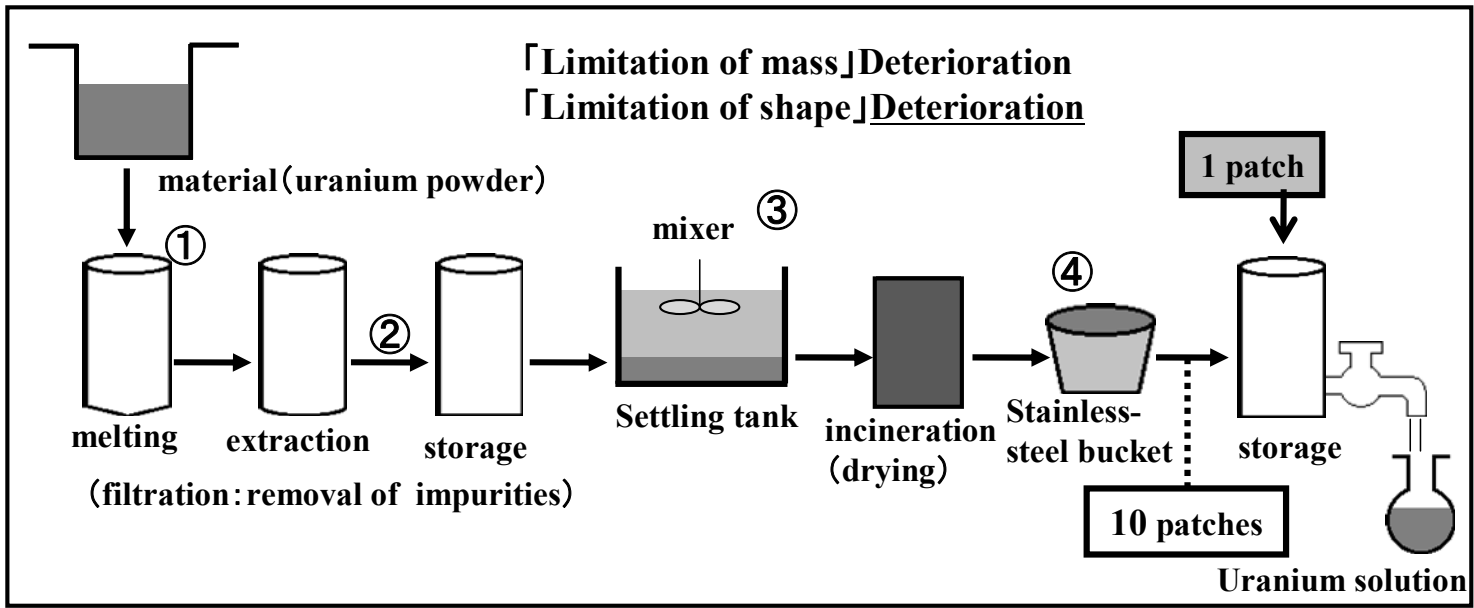

4th alteration of manufacturing process

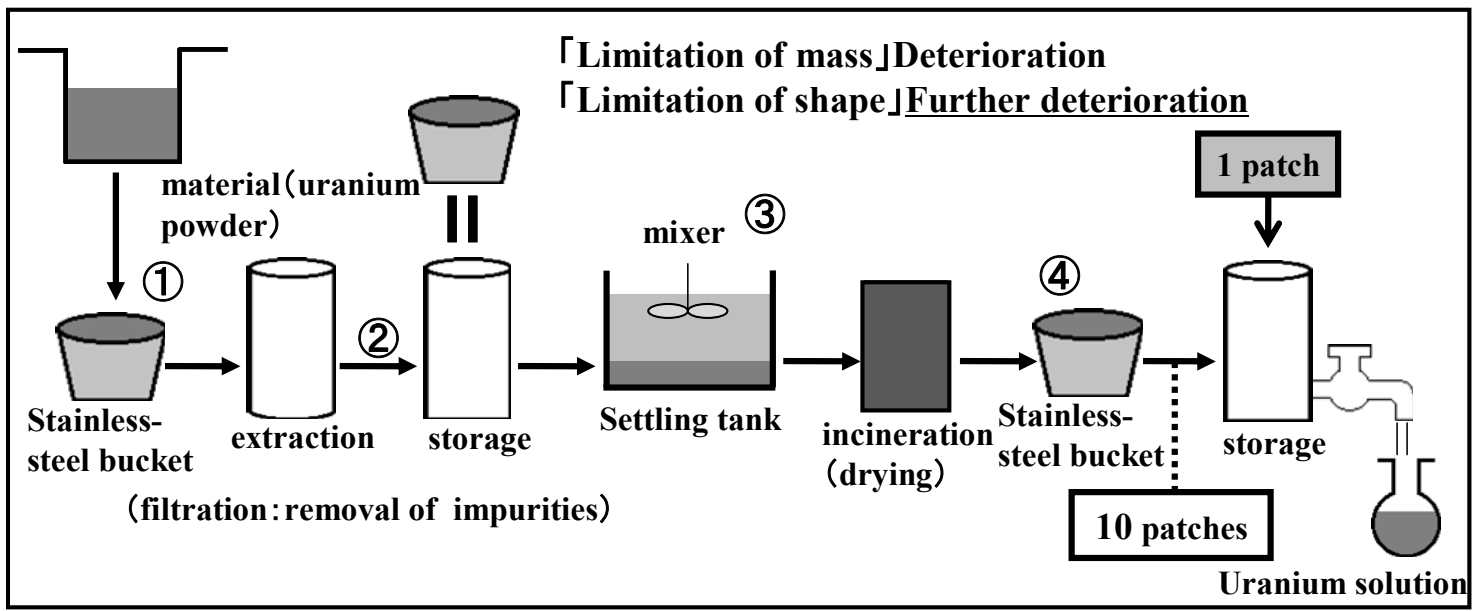

5th alteration of manufacturing process

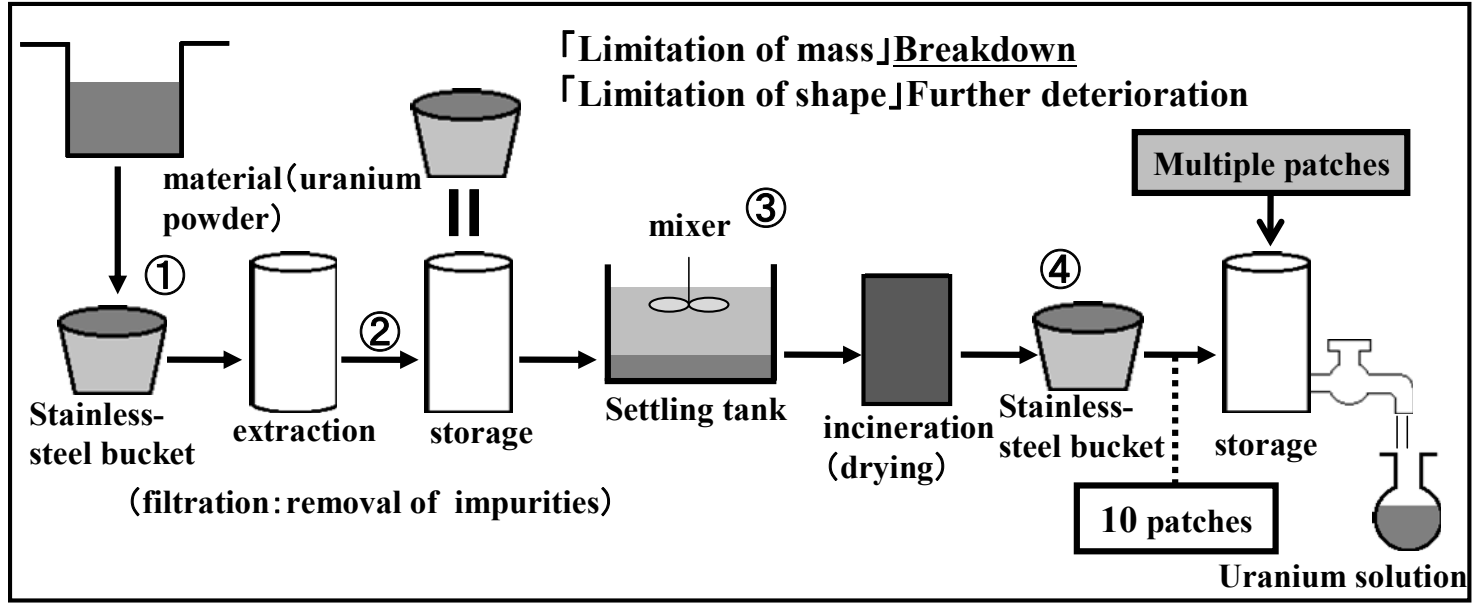

(Figure 4 to be continued) 
6th alteration of manufacturing process

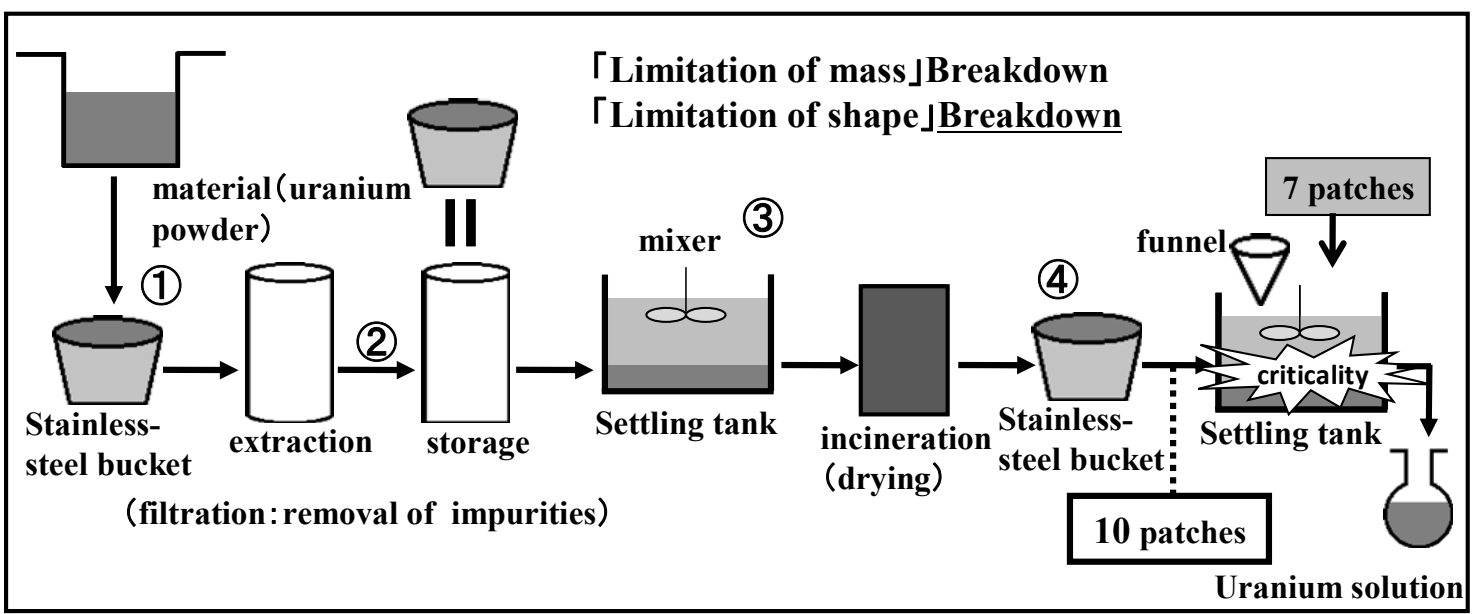

Figure 4. Multiple alteration of manufacturing process in critical mass accident at JCO Tokai Works Test Facility uranium processing plant.

In the field of behavioral ethics, it has been shown that we tend to overlook others' unethical behavior when it occurs gradually than when it occurs at a burst (Gino \& Bazerman, 2009). We are less likely to take others unethical behavior seriously when it eroded gradually over time rather than when it eroded in one gulp. Such a phenomenon is known as slippery-slope effect or gradual escalation of unethical behavior. In such a situation, we tend to weigh the ethicality and the economic incentive in the balance, and gradually escalate unethical behaviors as shown in Figure 4.

In safety management, we are forced to be confronted with such a situation where we weigh the safety (ethicality) and the efficiency (economic incentive). Although we thoroughly understand that it is essential to observe safety rules, we gradually escalate and erode safety ethics or rules and pursuit efficiency. Although we feel less guilty when violating a safety rule gradually instead of violating a safety rule at a time, such a slippery-slope effect leads to a blind spot of safety, and unintended crucial accidents like critical mass accident at the uranium processing plant of JCO Tokai Works Test Facility (Chiles, 2002). The slippery-slope effect pointed out by Gino and Bazerman (2009) can also be interpreted from the viewpoint of risk taking. Gradual violation of a safety rule instead of violating a safety rule at a time paralyzes our ethical attitude towards safety and makes us feel less guilty of violating a safety rule or ethics. Gradual escalation of risk taking behavior dilutes more our attitude towards safety and makes us underestimate a risk than taking a risk at a time.

\section{Conclusions}

As a result of exploring the relationship between the diversification of risk (accepting (taking) the total risk gradually by dividing it a few trials) and the risk assessment, the participants tended to underestimate the total risk when the total risk was divided to a few risk-taking behaviors than when the total risk was taken at a time.

Future research should discuss further how the finding that the gradual escalation of risk taking behavior can be more readily accepted than risk taking in one and at a time should be effectively made use of to prevent violation-based crucial accidents such as mentioned above from occurring. 


\section{References}

Altman, M. A. (2012). Behavioral economics for dummies. John Wiley \& Sons Canada, Ltd.

Angner, E. (2012). A course in behavioral economics. Palgrave Macmillan.

Ariery, D. (2009). Predictably irrational-The hidden forces that shape our decisions. Harper.

Ariery, D. (2010). The upside of irrationality -The unexpected benefits of defying logic at work and at home. Harper.

Ariery, D. (2012). The (honest) truth about dishonesty - How we lie to everyone - Especially ourselves. Harper.

Bazerman, M. H., \& Moore, D. A. (2001). Judgment in managerial decision making. Harvard University Press.

Bazerman, M. H., \& Tenbrunsel, A. E. (2012). Blind spot: Why we fail to do what's right and what to do about it. Princeton University Press.

Chiles, J. R. (2002). Inviting disaster—Lessons from the edge of technology. Harper, N.Y.: New York.

Gino, F., \& Bazerman, M. H. (2009). When misconduct goes unnoticed: The acceptability of gradual erosion in others' unethical behavior. Journal of Social Psychology, 45, 708-719.

Hosmer, L. T. (1987). The institutionalization of unethical behavior. Journal of Business Ethics, 6(6), 439-447.

Kahneman, D. (2011). Thinking, fast and slow. Penguin Books.

Kahneman, D., \& Tversky, A. (1984). Choices, values, and frames. American Psychologist, 39(4), 341-350.

Moore, D. A., Tetlock, P. E., Tanlu, L., \& Bazerman, M. H. (2006). Conflicts of interest and the case of auditor independence: Moral seduction and strategic issue cycling. Academy of Management Review, 31(1), 10-29.

Murata, A., \& Nakamura, T. (2014). Basic study on prevention of human error-How cognitive biases distort decision making and lead to crucial accidents. Proc. of AHFE 2014, 136-141.

Tversky, A., \& Kahneman, D. (1974). Judgment under uncertainty: Heuristics and biases. Science, 185, 1124-1131. 